\title{
Enhancement of the entanglement generation via randomly perturbed series of external pulses in a nonlinear Bose-Hubbard dimer
}

\author{
J. K. Kalaga · A. Kowalewska-Kudłaszyk • M. W. Jarosik • R. Szczȩśniak • W. Leoński®
}

Received: 9 March 2019 / Accepted: 16 June 2019 / Published online: 27 June 2019

(C) The Author(s) 2019

\begin{abstract}
We discuss a model of a short BoseHubbard dimer involving two anharmonic quantum oscillators mutually coupled with the use of the linear interaction and additionally driven by a series of ultra-short external pulses. We show that under some conditions the system behaves as a two-qubit one, and contrary to its continuously driven counterpart can be a source of maximally entangled states (MES). We discuss the cases when the system is excited only in one mode and in both ones, and additionally when the cross-Kerr-type interaction is present or omitted. We show that the generation of maximally (or almost maximally) entangled states depends on the time of free evolution of oscillators and hence, the proper steer-
\end{abstract}

A. Kowalewska-Kudłaszyk

Nonlinear Optics Division, Faculty of Physics, Adam Mickiewicz University, Uniwersytetu Poznańskiego 2, 61-614 Poznań, Poland

J. K. Kalaga · W. Leoński ( $\square)$

Quantum Optics and Engineering Division, Institute of Physics, University of Zielona Góra, Prof. Z. Szafrana 4a, 65-516 Zielona Góra, Poland

e-mail: w.leonski@if.uz.zgora.pl

J. K. Kalaga · W. Leoński

Joint Laboratory of Optics of Palacký University and Institute of Physics of CAS, Faculty of Science, Palacký

University, 17. listopadu 12, 77146 Olomouc,

Czech Republic

M. W. Jarosik · R. Szczȩśniak

Institute of Physics, Czȩstochowa University of

Technology, Armii Krajowej 19, 42-200 Częstochowa, Poland ing of the changes of the time between subsequent pulses can increase the effectiveness of MES generation. Moreover, we show the influence of the timing between two series of pulses (corresponding to the two modes) on such a process. What is relevant, thanks to the presence of additional randomness in the timings, the states with stronger entanglement can be achieved than for the situation when the time between consecutive pulses remains ideally fixed.

Keywords Bose-Hubbard system · Nonlinear oscillator - Kerr-like nonlinearity - Quantum entanglement . Bell states

\section{Introduction}

In recent years, the possibility of creation and manipulation of quantum states of various characteristic has been studied very intensively. Primarily, an important research direction was orientated to the generation of entanglement in quantum systems, which is especially relevant for the development of the broadly understood quantum information theory and its applications. Thus, maximally (or in real systems, almost maximally) entangled states can be implemented in the field of quantum communication, quantum cryptography, or quantum computations (for instance, see [1-10] and the references quoted therein). Maximally entangled states are also necessary for successful quantum teleportation experiments $[3,11,12]$. Another valid group of prob- 
lems in which entangled states are applied is related to the flow of quantum entanglement and information [13-15]. The nature of such flow, due to its quantum character, considerably differs from that appearing in classical systems (for instance, see [16-19]).

It should be emphasized that entangled states can be applied in various high-tech applications in the future. For instance, they can be used establishing the money system based on quantum technologies, highly protected against the forgery [20]. Another group of possible applications of the entangled states is related to the proposals of quantum internet [21]. In particular, quite recently, the experiment which can lead to the construction of quantum routers was performed and presented in [22]. At this point, one should mention the successful quantum teleportation from the surface of the Earth to the satellite [23], which opens new perspectives in the development of quantum technologies. Therefore, finding effective methods, and improving those already existing, allowing to produce various kinds of entangled states became one of the most substantial challenges in contemporary quantum physics. It was the primary motivation in our work presented here.

In this paper, we shall show how to improve the generation of entangled states not only by proper tuning of the parameters describing model discussed here but also by adding some randomness to the process of external excitation. In particular, we will concentrate on the Bose-Hubbard model involving two nonlinear oscillators of Kerr-type characteristic (the BoseHubbard dimmer), which are excited by a series of ultra-short pulses in one or two of the modes. In quantum optics, such systems are usually referred to as nonlinear coupler and were proposed in 1982 by Jensen [24] and Mă̌er [25]. Kerr-like couplers were intensively investigated (see [26-28] and the references quoted therein), and numerous features in such systems have been observed and discussed, including subPoissonian and squeezed light [29-34] or quantum entanglement generation [35-38]. It should be emphasized that the Bose-Hubbard model we propose is general enough to describe various physical systems in which the phenomenon of quantum entanglement can be observed. For instance, entangled states can appear in Bose-Einstein condensates [39-41], quantum dots $[42,43]$, trapped ions [44], atoms inside an optical cavity [45,46] and in many others [47-52].

Usually, the systems applied as sources of the maximally entangled states are those in which quantum evo- lution remains closed within a set of the states defined in a finite-dimensional Hilbert space. Such systems are referred to as quantum scissors [53] (when they consist of linear or nonlinear elements, they are linear (LQS) or nonlinear quantum scissors (NQS), respectively) and lead to the appearance of so-called photon (phonon) blockade effects [54-56].

It should be pointed out that the simplest models involving only one single Kerr-type oscillator can also lead to the appearance of diverse, compelling phenomena and effects, and were a subject of many papers. For example, for such nonlinear models generation of various quantum states of the electromagnetic (EM) field can appear [57-62]. In particular, the kicked anharmonic oscillator model was discussed as a potential source of Fock states-one-photon states [63]. Next, it was shown that the same system could lead to the generation of the finite-dimensional coherent states [64]. Moreover, depending on the time of the free system's evolution (time between two subsequent external pulses), a superposition of only two Fock states or superposition of a much larger number of Fock states can be achieved $[65,66]$. Such models were also considered in other contexts, such as the ergodicity of systems $[67,68]$, quantum-classical correspondence $[69,70]$ or quantum chaotic behavior [71-78].

As it was mentioned above, quantum entanglement can be generated in Bose-Hubbard systems involving at least two nonlinear oscillators. The discussion of such features was presented in [35-38]. In those studies, the time between two subsequent pulses was arbitrarily chosen and assumed to be constant. This fact is a good motivation to study the relations between the effectiveness of the generation of maximally (or almost maximally) entangled states and the choice of time when the oscillators evolve freely. The situation becomes even more interesting when we assume the excitations in two modes. Thus, the main aim of this paper is to show not only that our model can be treated as NQS but primarily that changes in the time of the free evolution of the oscillator (or two oscillators) considerably changes the degree of the entanglement produced by the system. We shall discuss how such time duration and the relation between the times corresponding to the two oscillators can affect the process of MES generation, and how it differs from the cases when continuous pumping is assumed. What is the most interesting, we will show that some random variances of those times can improve the process spoiled by not ideally chosen 
time distance between two subsequent pulses. The last aspect seems especially interesting as it is challenging to maintain precisely stable timing for the pulses in the real experimental situations. Moreover, we compare two situations-when the cross-Kerr-type coupling is present and is neglected.

The paper is organized as follows. In Sect. 2, we define the system consisting of two anharmonic quantum oscillators coupled to each other and driven (in one or two modes) by an external field. Such external field is assumed to be in the form of series of very short pulses. Next, we concentrate on the system's evolution, especially on the possibility of the MES generation. In particular, Sect. 3 discusses the case when the system is excited only in one mode. We derive there the formulas describing the probability amplitudes corresponding to the two situations: when the cross-Kerr-type interaction is present and when is omitted. We study there the possibility of the creation of MES and next compare the effectiveness of such process with that appearing in the continuously excited models. We check how the duration of the time of the free evolution (times between two pulses) influences the effect of the entanglement generation. In Sects. 4 and 5, we concentrate on the case when both oscillators are excited by the pulsed fields. Finally, in Sect. 6 we discuss the influence of random changes in times between subsequent pulses (in both modes) on the effectiveness of the creation of entangled states. We show that such randomness can improve the efficiency of the entanglement generation.

All numerical calculations were performed with the application of the MATLAB ${ }^{\circledR}$ software. Also, the figures containing the plots were prepared using the MATLAB $^{\circledR}$ package.

\section{The model}

Our system comprises two identical anharmonic quantum oscillators mutually coupled by linear interaction. Additionally, the external pulsed EM field excites one or two oscillators. Such excitations are modeled by Dirac-delta functions. For the cases when both oscillators are influenced by the external field, we deal with two situations-the oscillators are driven synchronically or in an unsynchronous way.

Our previous considerations, presented in [79] and concerning the possibility of formation of the 2-qubit MES in the similar system, were devoted to the sit-

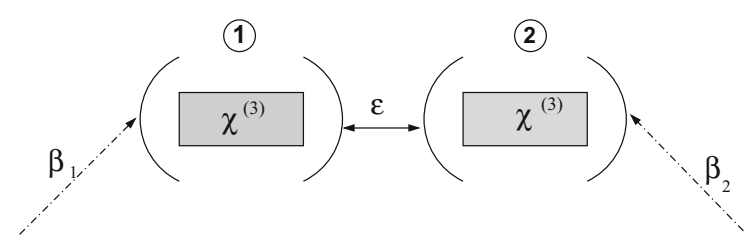

Fig. 1 The Bose-Hubbard model considered here comprising two interacting nonlinear oscillators (Kerr-type), excited by the train of external pulses

uation when only one mode pumping was present. It was shown there that for the appropriate choice of the parameters describing interactions present in the system, the system not only behaves as NQS but becomes a source of Bell-like MES with high, although not perfect, probability.

We can describe the system considered here by the following Hamiltonian:

$$
\begin{aligned}
\hat{H}_{\text {eff }}= & \frac{\chi_{1}}{2}\left(\hat{a}_{1}^{\dagger}\right)^{2} \hat{a}_{1}^{2}+\frac{\chi_{2}}{2}\left(\hat{a}_{2}^{\dagger}\right)^{2} \hat{a}_{2}^{2} \\
& +\chi_{12} \hat{a}_{1}^{\dagger} \hat{a}_{1} \hat{a}_{2}^{\dagger} \hat{a}_{2}+\varepsilon \hat{a}_{1}^{\dagger} \hat{a}_{2}+\varepsilon^{\star} \hat{a}_{1} \hat{a}_{2}^{\dagger},
\end{aligned}
$$

whereas the external excitation(s) is(are) described by:

$\hat{H}_{i}=\left(\beta_{1} \hat{a}_{i}^{+}+\beta_{i}^{\star} \hat{a}_{i}\right) \sum_{k}^{\infty} \delta\left(t-k T_{i}\right), \quad i=\{1,2\}$,

where $\hat{a}_{i}\left(\hat{a}_{i}^{+}\right)$are the annihilation (creation) operators for the $i$-th $(i=\{1,2\})$ oscillator's modes, whereas $\chi_{i}\left(\chi_{12}\right)$ are the $3^{r d}$-order susceptibilities describing self- and cross-action processes, respectively. For the simplicity, we assume that oscillators are identical, i.e., $\chi_{1}=\chi_{2}=\chi$. The parameters $\varepsilon, \beta_{i}$ describe the mutual and external interactions, respectively. The time $T_{i}$ denotes the time between two subsequent pulses in $i$-th mode (Fig. 1).

When considering the whole time evolution of the system which is excited by ultra-short pulses, one can distinguish two stages/kinds of the evolution. The first of them corresponds to the action of a single external pulse. For such a case, the energy of the system changes. The second stage corresponds to the periods between two subsequent pulses. If the damping processes are neglected, the total energy of the oscillatory system does not change during those times. Since in the present paper, only quantum states formation with the absence of damping processes is considered (we will not include the influence of the external environment), the solutions of the Schrödinger equations will be considered. The wave-function describing the evolution of the system 
of two oscillators can be obtained by the application of quantum mapping procedure [70].

Thus, we define the unitary evolution operators corresponding to the above-mentioned two stages of the evolution. First, the operator governs the action of external pulse in one mode we define as

$\hat{U}_{K}=\exp \left(-i\left(\beta_{i} \hat{a}_{i}^{\dagger}+\beta_{i}^{*} \hat{a}_{i}\right)\right)$,

whereas the operator describing pulsed two-mode excitations is defined as

$\hat{U}_{K}=\exp \left(-i\left(\beta_{1} \hat{a}_{1}^{\dagger}+\beta_{1}^{*} \hat{a}_{1}+\beta_{2} \hat{a}_{2}^{\dagger}+\beta_{2}^{*} \hat{a}_{2}\right)\right)$.

Next, we define the operator corresponding to the "free" evolution of coupled nonlinear oscillators during the time $T$ as

$\hat{U}_{N L}=\exp \left(-i\left(\hat{H}_{\mathrm{eff}} T\right)\right)$.

Such defined operators multiply acting in the appropriate order on the initial state give the state after each external pulse.

After the action of the $k$-th pulses, it can be written in a general form:

$\left|\Psi_{k}\right\rangle=\left(\hat{U}_{N L} \hat{U}_{K}\right)^{k}\left|\Psi_{0}\right\rangle$

It is valid for both cases-excitations in a single mode and excitations in both modes. It should be pointed out that the relation (6) can be applied when two trains of pulses act simultaneously at the same moments of time. When one train of excitations is shifted in time compared to the second, the mapping procedure gives:

$\left|\Psi_{k}\right\rangle=\left(\hat{U}_{N L_{2}} \hat{U}_{K_{2}} \hat{U}_{N L_{1}} \hat{U}_{K_{1}}\right)^{k}\left|\Psi_{0}\right\rangle$.

The unitary evolution operators $U_{N L}\left(U_{N L_{i}}\right)$ and $U_{K}\left(U_{K_{i}}\right)$ can be expressed with the use of parameters describing the system as:

$\hat{U}_{N L_{i}}=\exp \left(-i \hat{H}_{\mathrm{eff}} T_{i}\right)$

$\hat{U}_{K}=\exp \left[-i\left(\beta_{1} \hat{a}_{1}^{+}+\beta_{1}^{\star} \hat{a}_{1}+\beta_{2} \hat{a}_{2}^{+}+\beta_{2}^{\star} \hat{a}_{2}\right)\right]$

$\hat{U}_{N L}=\exp \left(-i \hat{H}_{\text {eff }} T\right)$

$\hat{U}_{K_{1}}=\hat{U}_{K}$ with $\beta_{2}=0$ and $\hat{U}_{K_{2}}=\hat{U}_{K}$ with $\beta_{1}=0$

We assume here that the mutual interaction of the oscillators, described by $\varepsilon$, is considerably smaller than the nonlinearity parameter $\chi_{i}$ of any of the oscillators. Additionally, the value of $\varepsilon$ is also lower than (or at least equal to) the external interaction strength $\beta$ so that the dominant parameter of the system is the thirdorder susceptibility $\chi$. Usually, in optical systems, it is expected that Kerr nonlinearity is weak. However, there are numerous examples of physical models in which the Kerr-type nonlinearities are sufficiently strong - for example, see the systems in which electromagnetically induced transparency (EIT) effects in atomic configurations appear [80]. We would like to emphasize that our proposal more likely should be referred to the group of systems (optical, optomechanical, etc.) in which the Kerr nonlinearity appears, for example, as a result of strong atom-cavity mode or qubit-nanoresonator field interactions. Such systems can be described by the effective Hamiltonians which involve Kerr-like nonlinearities. In consequence, the values of the effective third-order susceptibility parameters can be externally controlled and one can fulfill the conditions assumed in the present considerations.

\section{Entanglement generation in the system with single-mode excitation}

In further considerations, we are mainly interested in the generation of entangled quantum states, especially MES. Therefore, for the analysis of the degree of entanglement obtained in the system, we shall use as the entanglement measure, the negativity. It is defined as [81,82]:

$N(\rho)=\frac{1}{2} \sum_{j}\left|\lambda_{j}\right|-\lambda_{j}$,

where $\lambda_{j}$ is $j$-th eigenvalue of the matrix $\rho^{T_{i}}$-a partially (with respect to one of the subsystems $i=1$ or 2 ) transposed density matrix $\rho$ describing the whole system. The negativity can be treated as a pure measure of the entanglement when it is applied to two-qubit or qubit-qutrit systems. For such cases, it can differentiate between entangled and not entangled states-it is equal to the unity for MES and zero for the separable states.

As a starting point in the detailed analysis of the formation of entangled states within the system of two nonlinear oscillators, we choose a single-mode excitation case. This problem was already partially presented 
in [53]. In presented here considerations we shall compare the results corresponding to the models assuming continuous external excitations (discussed in many aspects in [26] or in general in [53]) to those corresponding to our system excited by a train of pulses.

In the review paper [53] devoted to the formation of truncated quantum states in various nonlinear systems, we have shown that when coupled anharmonic oscillators are excited by ultra-short pulses, the system's evolution can be restricted to a few states only. In [79], when only one of the coupled oscillators was externally driven, we have shown that despite the continual excitations by ultra-short pulses, only four resonant states are populated and in fact, such system behaves as NQS. In consequence, the probability amplitudes corresponding to the populated states $|0\rangle|0\rangle,|0\rangle|1\rangle,|1\rangle|0\rangle$ and $|1\rangle|1\rangle$ show oscillatory behavior with characteristic frequencies describing their evolution [79].

First, we shall concentrate on the situation when cross-coupling is omitted $\left(\chi_{12}=0\right)$. Thus, the equations describing the time evolution of the probability amplitudes are given as in [79] by:

$$
\begin{aligned}
c_{00}(k)= & \frac{1}{2 T \Omega_{0} \varepsilon}\left[\left(2 \beta^{2}-\Omega_{1}^{2}\right) \cos \left(\frac{k \Omega_{2}}{\sqrt{2}}\right)\right. \\
& \left.-\left(2 \beta^{2}-\Omega_{2}^{2}\right) \cos \left(\frac{k \Omega_{1}}{\sqrt{2}}\right)\right] \quad(10 \mathrm{a}) \\
c_{01}(k)= & \frac{\beta}{\Omega_{0}}\left[\cos \left(\frac{k \Omega_{2}}{\sqrt{2}}\right)-\cos \left(\frac{k \Omega_{1}}{\sqrt{2}}\right)\right] \quad(10 \mathrm{~b}) \\
c_{10}(k)= & \frac{i \beta}{\sqrt{2} T \varepsilon \Omega_{0} \Omega_{1} \Omega_{2}} \\
& \times\left[-2\left(\varepsilon^{2} T^{2}+\beta^{2}\right) \Omega_{1} \sin \left(\frac{k \Omega_{2}}{\sqrt{2}}\right)\right. \\
& \left.+\varepsilon T\left(\varepsilon T-\Omega_{0}\right) \Omega_{2} \sin \left(\frac{k \Omega_{1}}{\sqrt{2}}\right)+\Omega_{1}^{2}\right] \\
c_{11}(k)= & i \frac{\sqrt{2} \beta^{2}}{\Omega_{0}}\left[\frac{1}{\Omega_{1}} \sin \left(\frac{k \Omega_{1}}{\sqrt{2}}\right)-\frac{1}{\Omega_{2}} \sin \left(\frac{k \Omega_{2}}{\sqrt{2}}\right)\right],
\end{aligned}
$$

and the frequencies: $\Omega_{0}=\sqrt{4 \beta^{2}+\varepsilon^{2} T^{2}}, \Omega_{1}=$ $\sqrt{2 \beta^{2}+\varepsilon^{2} T^{2}-\varepsilon T \Omega_{0}}$ and $\Omega_{2}=\sqrt{2 \beta^{2}+\varepsilon^{2} T^{2}+\varepsilon T \Omega_{0}}$.
When $\chi_{12} \neq 0$, the equations for the probability amplitudes take the following much simpler form:

$$
\begin{aligned}
& c_{00}(k)=\frac{1}{\Omega^{2}}\left[\varepsilon^{2} T^{2}+\beta^{2} \cos (k \Omega)\right] \\
& c_{01}(k)=\frac{\beta \varepsilon T}{\Omega^{2}}[-1+\cos (k \Omega)] \\
& c_{10}(k)=-i \frac{\beta}{\Omega} \sin (k \Omega),
\end{aligned}
$$

where $\Omega=\sqrt{\beta^{2}+\varepsilon^{2} T^{2}}$. We see that for such a case only three resonant states are populated, and oscillations are governed by a single frequency depending mainly on the strengths of the excitation and interactions between oscillators. In Fig. 2, we present the analysis of the system externally driven in one mode. We plotted only the maximal values of the negativity $N_{0110}$ for MES defined in the space spanned over the group of two-mode states $\{|0\rangle|0\rangle,|0\rangle|1\rangle,|1\rangle|0\rangle,|1\rangle|1\rangle\}$ describing of the whole system. We have chosen two exemplary values of the relations between external $(\beta)$ and internal $(\varepsilon)$ interactions. Moreover, we present here two situations: when $\chi_{12}=0$ (Fig. 2a, c) and that corresponding to $\chi_{12}=1$ (Fig. $\left.2 b, d\right)$.

In each of the cases, we can easily find the ranges of the values of parameters for which the strongest possible entanglement in the two-qubit system can be achieved. In general, when only self-Kerr-type nonlinearities are present in the system, no significant differences between the maximal values of $N_{0110}$ for the pulsed and continuous excitations can be noticed. The maximal values of the negativity which can be achieved are listed in Table 1. The time dependencies of the negativity slightly differ between each other, but the results are comparable. However, when additional cross-Kerrtype interaction is added to the model, the situation changes significantly, and hence, one can obtain much higher values of the negativity for pulsed excitations in a wide range of the time $T$ (see in Fig. 2d where external excitation strength is dominant over the mutual coupling between the oscillators). That problem was also pointed out in [79], but it was discussed there only for one arbitrarily chosen set of parameters. The analysis performed here allows us identifying much broader ranges of the values of the parameters for which pulsed excitations are more efficient in producing MES.

Additionally, a large decrease in the negativity can be seen for the extrema-for small and large values of $T$ (time of free evolution). Especially, when $\chi_{12}=0$ 

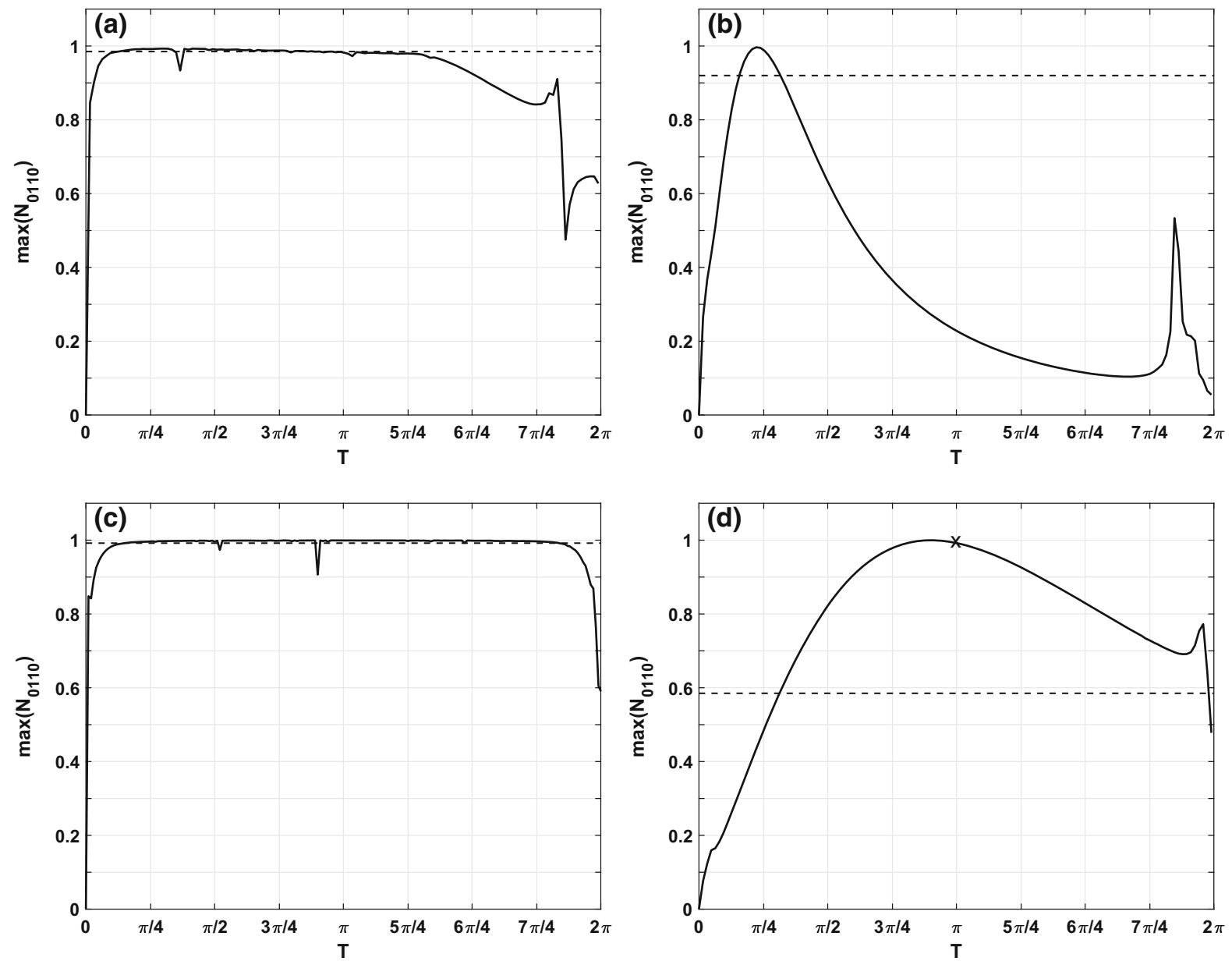

Fig. 2 Maximal values of the negativity $N_{0110}$ when only one oscillator is excited (solid lines) versus the time $T$ of the free evolution of both oscillators: a $\beta / \chi=\epsilon / \chi=1 / 25, \chi_{a b}=0$; b $\beta / \chi=\epsilon / \chi=1 / 25, \chi_{a b}=1 ;$ c $\beta / \chi=1 / 25, \epsilon / \chi=$ $1 / 100, \chi_{a b}=0 ; \mathbf{d} \beta / \chi=1 / 25, \epsilon / \chi=1 / 100, \chi_{a b}=1$. Addi-

tionally, the reference dashed line corresponding to the value of $N_{0110}$ for the same model but with continuous driving in one mode is plotted. The nonlinearity parameter $\chi=1$. Mark " $x$ " corresponds to the results discussed in [79]

Table 1 The maximal values of the negativity which can be gained for the single-mode excitation model

\begin{tabular}{lll}
\hline & The system with continuous driving & The system with pulsed excitations \\
\hline$\beta / \chi=\epsilon / \chi=1 / 25 \chi_{a b}=0$ & $\max N_{0110}=0.9923$ & $\max N_{0110}=0.9937$ \\
$\beta / \chi=\epsilon / \chi=1 / 25 \chi_{a b}=1$ & $\max N_{0110}=0.9176$ & $\max N_{0110}=0.9965$ \\
$\beta / \chi=1 / 25 \epsilon / \chi=1 / 100 \chi_{a b}=0$ & $\max N_{0110}=0.9977$ & $\max N_{0110}=0.9994$ \\
$\beta / \chi=1 / 25 \epsilon / \chi=1 / 100 \chi_{a b}=1$ & $\max N_{0110}=0.5935$ & $\max N_{0110}=0.9995$ \\
\hline
\end{tabular}

(see Fig. 2a, c). Such a decrease is an effect of the fact that for the considered here values of $T$, a larger group of two-mode states is populated in the evolution of the whole system. Apart from the states belonging to the set $\mathfrak{B}=\{|0\rangle|0\rangle,|0\rangle|1\rangle,|1\rangle|0\rangle,|1\rangle|1\rangle\}$, which are responsible for obtaining entanglement measured by $N_{0110}$, there are also nonzero probabilities corresponding to the states $|0\rangle|2\rangle$ and $|2\rangle|0\rangle$. Although correlations 
responsible for the entanglement formation between those two states are negligible, nonzero population of the states which do not belong to $\mathfrak{B}$ is the main reason for not having the maximum entanglement $N_{0110}$ for the cases of the short (smaller than $\approx 0.1 \pi$ ) and long (close to $2 \pi$ ) time $T$. For other values of $T$, the analysis of $N_{0110}$ shows that the only two-mode states which are populated in the evolution of the whole system are the states $\{|0\rangle|0\rangle,|0\rangle|1\rangle,|1\rangle|0\rangle,|1\rangle|1\rangle\}$.

We would also like to emphasize that it is evident from the analysis presented in Fig. 2c, $d$ that there is a much broader range of parameters for which the states which are close to the Bell-like ones are generated when using pulsed excitations rather than using continuous driving.

In [79], we have shown that the system of coupled oscillators, which initially are in the vacuum state, excited in one mode only, can be a source of the Belllike states of the form:

$$
\begin{aligned}
|B\rangle_{1,2} & =\frac{1}{\sqrt{2}}(|0\rangle|0\rangle \pm i|1\rangle|1\rangle) \\
|B\rangle_{3,4} & =\frac{1}{\sqrt{2}}(|0\rangle|1\rangle \pm i|1\rangle|0\rangle)
\end{aligned}
$$

Depending on the value of the cross-interaction, one can obtain $|B\rangle_{1}$ state (for $\chi_{12}=0$ and $\varepsilon<\beta$ ) or $|B\rangle_{3,4}$ (for $\chi_{12} \neq 0$ and $\varepsilon<\beta$ ) with the probability close to the unity, whereas the state $|B\rangle_{2}$ can be produced with a smaller probability.

\section{Synchronous excitations}

Here, we shall concentrate on the cases when the pulses in both modes act on the system synchronously - at the same moments of time. For such situation, the times between two subsequent pulses are identical for the two pumping modes-we assume here that $T=\pi$. In consequence, the quantum mapping procedure can be described by the equation (6). Moreover, for simplicity, we assume that the excitations of both oscillatory modes are of the same strengths, i.e., $\beta_{1}=\beta_{2}$.

Depending on the fact whether the cross-Kerr-type interaction is present or not, different entangled twoqubit states can be produced. Thus, when only self-Kerr nonlinearities are included in our model, the abovementioned Bell-like states (see Eqs. 12a and 12b) can be built. However, when we also include the crossnonlinearity, only one of such type MES is created:

$|B\rangle=\frac{1}{\sqrt{2}}(|0\rangle|1\rangle+|1\rangle|0\rangle)$.

Figure 3 depicts the dependence of the maximal value of negativity max $N_{0110}$ on the time $T$ of free evolution between two subsequent external pulses, where the value of $N_{0110}$ shows how strong entanglement appears in the subspace defined by the states $\{|0\rangle|0\rangle,|0\rangle|1\rangle,|1\rangle|0\rangle,|1\rangle|1\rangle\}$. Here, the same as in Fig. 2, we discuss two representative values of the ratio $\beta / \chi=\varepsilon / \chi$ describing the relation between the internal and external interactions. Moreover, we also show the results for the two situations - when the crossinteraction is omitted or included in the model. We see that for the considered here values of $\beta / \chi=\varepsilon / \chi$, MES can be created for the broad ranges of the value of $T$. Such a process is more pronounced when the cross-Kerr-type nonlinearity is neglected (Fig. 3a, c). Therefore, when the system involves only the self-Kerr nonlinearities, the entanglement generation is not so much dependent on the careful choice of $T$-we see only tiny changes in $\max \left(N_{0110}\right)$ for the broad range of $T$. For $\pi / 4<T \lesssim 7 \pi / 4$ in both considered situations (Fig. 3a, c), MES can be created very effectively. Only for $T \lesssim \pi / 4$ and $T \gtrsim 7 \pi / 4$ such states would not be formed.

When $\chi_{12} \neq 0$ (Fig. 3b, d), we can also produce almost maximally entangled two-qubit states; however, the range of the values of $T$ when such states can appear is much smaller than for the previous case. In consequence, for such a situation we should more carefully control the free evolution time. What is relevant, for $\chi_{12}=0$ when the negativity $N_{0110}=1$, one of the states (12b), is generated, whereas for $\chi_{12} \neq 0$ when $N_{0110}=1$ the system is in the state (13).

For the better comparison of our results to those corresponding to the model assuming continuously excited system (in both modes), we denoted by dashed lines the reference values of the negativity when the external driving is continuous. It is seen that when we neglect cross-Kerr-type interaction, the maximal value of $N_{0110}$ is slightly closer to the unity (but still very close to 1) than the mentioned above reference values, for the broad range of $T$ (see Table 2).

When the cross-nonlinearity is included in the model (Fig. 3b, d), the differences between $\max N_{0110}$ and 

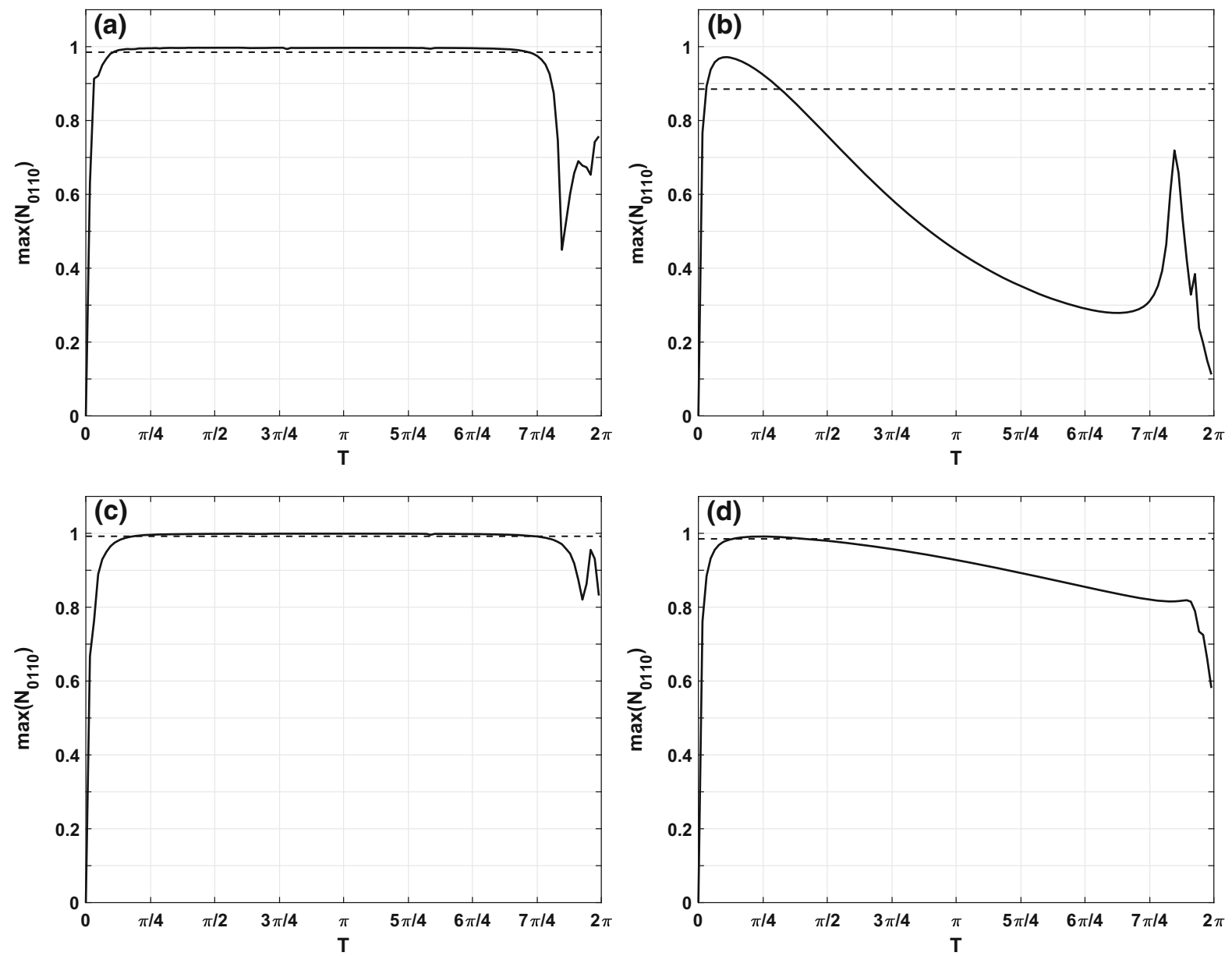

Fig. 3 Maximal values of the negativity $N_{0110}$ for the synchronously excited oscillators (solid lines) as a function of free evolution time $T: \mathbf{a} \beta / \chi=\epsilon / \chi=1 / 25, \chi_{a b}=0 ; \mathbf{b} \beta / \chi=$ $\epsilon / \chi=1 / 25, \chi_{a b}=1 ; \mathbf{c} \beta / \chi=1 / 25, \epsilon / \chi=1 / 100, \chi_{a b}=0$;

d $\beta / \chi=1 / 25, \epsilon / \chi=1 / 100, \chi_{a b}=1$. The dashed horizontal line denotes the reference value of the negativity for the continuously driven system. The values of other parameters are the same as in Fig. 2

Table 2 The maximal values of the negativity which can be gained for the model involving two-mode excitations

\begin{tabular}{lll}
\hline & The system with continuous driving & The system with pulsed excitations \\
\hline$\beta / \chi=\epsilon / \chi=1 / 25 \chi_{a b}=0$ & $\max N_{0110}=0.9963$ & $\max N_{0110}=0.9971$ \\
$\beta / \chi=\epsilon / \chi=1 / 25 \chi_{a b}=1$ & $\max N_{0110}=0.8848$ & $\max N_{0110}=0.9716$ \\
$\beta / \chi=1 / 25 \epsilon / \chi=1 / 100 \chi_{a b}=0$ & $\max N_{0110}=0.9975$ & $\max N_{0110}=0.9991$ \\
$\beta / \chi=1 / 25 \epsilon / \chi=1 / 100 \chi_{a b}=1$ & $\max N_{0110}=0.9899$ & $\max N_{0110}=0.9912$ \\
\hline
\end{tabular}

the reference values become more pronounced. This effect is especially apparent when the external excitations become comparable to the internal ones (Fig. 3b). Moreover, there is only a narrow range of the values of $T(\approx\langle\pi / 20 ; \pi / 3\rangle)$ for which pulsed system can generate entangled states characterized by the noticeable higher negativity than that continuously excited. In Fig. 3b, we see that for the pulsed system, when the time $T$ is appropriately chosen, the entangled states appearing in the system become more close to MES. 


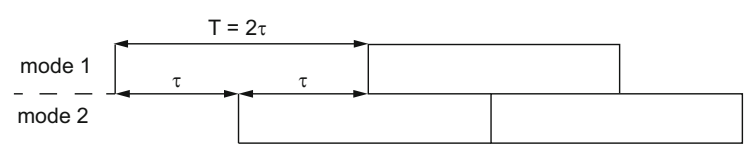

Fig. 4 The scheme, showing timings of asynchronous excitations

We see that although continuous excitation can lead to the formation of maximally or almost maximally entangled states (see, for example, [26]), the main advantage of the models driven by ultra-short pulses is that the latter enables obtaining the two-qubit entangled states characterized by higher negativity, especially when the interactions between the oscillators are weaker than external excitations. Such an effect becomes more pronounced when the cross-Kerr-type coupling is present, and consequently, the states (13), or (12b) can be formed. For the continuously excited model, such conditions lead to the formation of states of the type (12a) but with smaller efficiency. The discussion concerning such differences between the system driven by continuous excitation and that excited by external kicks can be found, for instance, in [79].

\section{Asynchronous excitations}

The next step in our considerations is to introduce the time shift between the trains of pulses corresponding to the two modes of the field. In consequence, the excitations of two oscillators will not act simultaneously, and hence, each of the oscillators will be excited at the different moments of time. In this subsection, we shall discuss the case when external excitations act alternately - one of the trains of pulses is shifted in relation to the second one, by the time $\tau$. For such a case, both sequences are characterized by the same $T=2 \tau$ describing the time of free evolution of oscillators. The scheme of excitations is plotted in Fig. 4. Here, we will concentrate on the influence of the time shift $\tau$ on the effectiveness of the entangled state's formation. It should be noted that the $\tau$ and $T$ remain constant during the whole system's evolution.

To perform the quantum mapping procedure, we will apply the parameter $\tau$ to the unitary operator (8a), where $T_{1}=T_{2}=\tau$ and then, the calculations will be done according to the formula (7). To check how the time-shift effect can influence the entangled state's generation, we will vary the value of $\tau$ and, analogously as for the previously discussed cases, will find the maximal values of the negativity max $N_{0110}$ for each value of $\tau$.

Figure 5 depicts two situations for which the relations between the two strengths of the interactions are $\beta=\varepsilon$ and $\beta<\varepsilon$ are assumed (Fig. 5a-d, respectively). Moreover, it presents the results for two models: one involving the cross-Kerr-type interaction (Fig. 5b, d) and the other omitting it (Fig. 5a, c).

From Fig. 5, we see that the creation of maximally (or almost maximally) entangled states considerably depends on the time $\tau$, and thus, the generation of entanglement can be sensible on the proper choice of the value of $\tau$.

When the cross-nonlinearity is omitted (Fig. 5a, c), MES can be formed. For such a case, $\max \left(N_{0110}\right)$ remains close to the unity for the broad range of values of $\tau$. Only when $\tau$ is close to zero or $\pi$, such states would not be generated. In consequence, such a situation seems to be the most promising in the effective forming of MES.

When $\chi_{a b} \neq 0$, the almost MES can be produced only for a narrow range of the values of $\tau$. Furthermore, with increasing $\tau$, the maximal value of achievable negativity decreases. Thus, for the systems involving cross-Kerr-type interaction, to produce MES, one should more carefully control the time $\tau$.

\section{Two-mode excitation with randomly disturbed free evolution's times}

The analysis of entanglement's evolution for the coupled nonlinear system driven by ultra-short pulses in both modes enabled us to find the optimal sets of parameters for the generation of maximally entangled two-qubit states. Primarily, we have shown that by the appropriate tuning of the values of the time shift $\tau$, and times of free-evolution $T$ one can improve the effectiveness of the process of creation of MES.

As we have shown in the previous section, there are situations when the formation of MES is not perfect or even impossible. Here, we shall show how adding some randomness to the non-ideally chosen values of the parameters presented in the previous section, can influence discussed here processes and, in consequence, improve the generation of entanglement.

Such, randomness appears in all real physical systems, as they are not perfect. For instance, if we are 

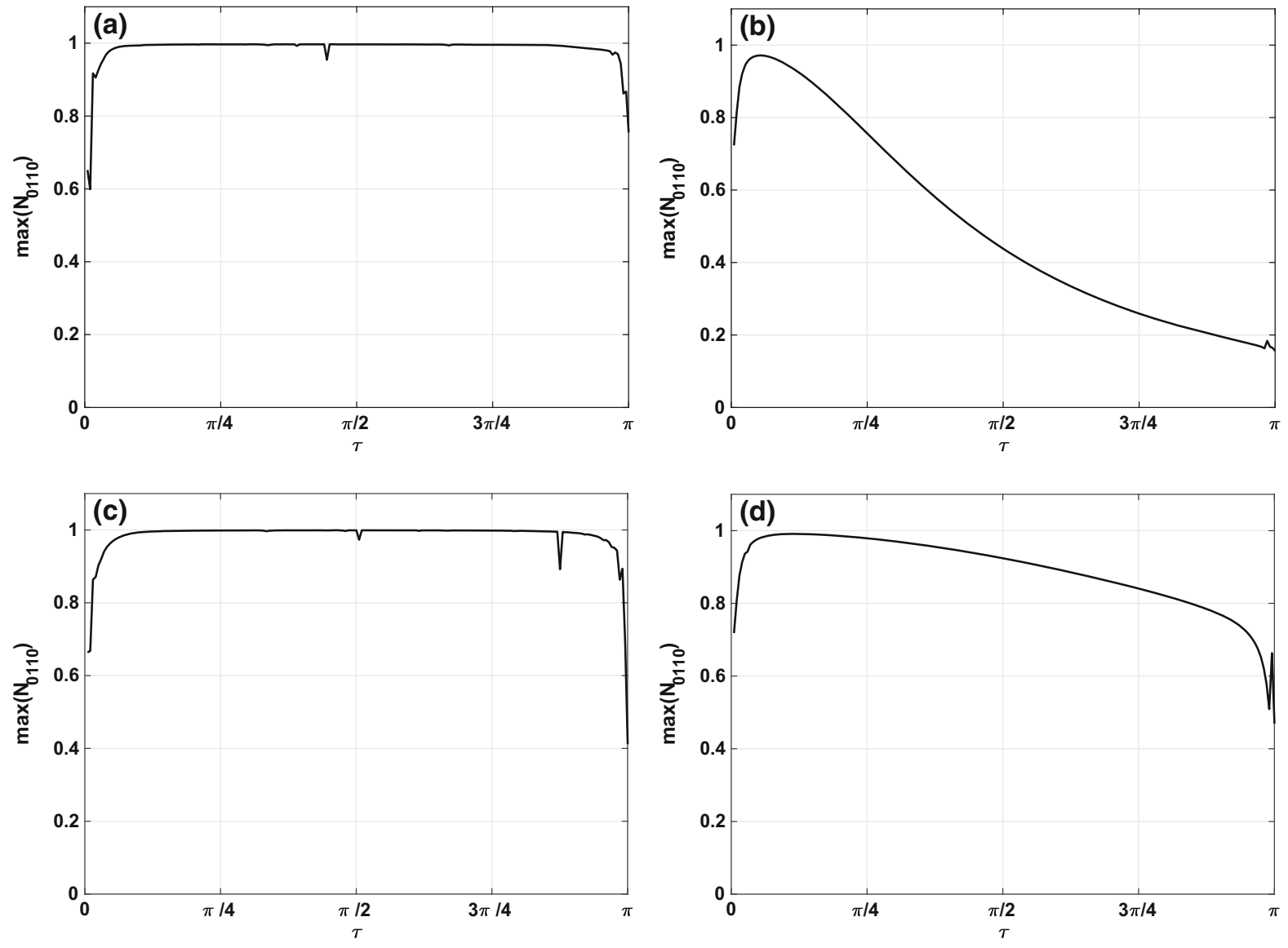

Fig. 5 Maximal values of the negativity $N_{0110}$ for the asynchronously excited oscillators versus $\tau$ : a $\beta / \chi=\epsilon / \chi=$ $1 / 25, \chi_{a b}=0 ; \mathbf{b} \beta / \chi=\epsilon / \chi=1 / 25, \chi_{a b}=1 ; \mathbf{c} \beta / \chi=$

$1 / 25, \epsilon / \chi=1 / 100, \chi_{a b}=0 ; \mathbf{d} \beta / \chi=1 / 25, \epsilon / \chi=$ $1 / 100, \chi_{a b}=1$. The remaining parameters are identical to those as shown in Fig. 2

dealing with lasers generating sequences of pulses, the time interval between two subsequent ones varies randomly from one pair of pulses to the next one-the timing jitter effect appears in all real laser systems. In consequence, pulse trains which are generated, e.g., in mode-locked lasers, exhibit some deviations of the temporal pulse positions [83]. Such variations originate, for instance, from noises present in the electronic oscillator driving the modulator within the laser [84$86]$ and allow manipulating with the statistical properties of timing. The analogous situation can be found in systems of the Q-switched lasers in which acoustooptic Q-switch is applied to influence the time between two subsequent pulses.

We can also propose a different physical situation in which we can randomly influence the train of pulses.

Namely, it is possible to apply a delay line in the optical path which contains a mirror randomly shifted by a piezo-element. Sending a random signal to the latter, we can modulate the length of the optical path of the laser signal and thus the timings of the pulse sequence.

Therefore, we shall modify our model by adding some random factor to the time $\tau$ characterizing the time of free evolution (see Fig. 4). As an example, we have chosen here such situations depicted in Fig. 5, for which the maximal value of the negativity was noticeably reduced. Such a situation appears when $\tau \simeq \pi$, and such values of $\tau$ are chosen for the two considered here cases corresponding to the two relations between $\beta$ and $\varepsilon$. 
Here, we modify the time $\tau$ by adding a random factor. Now, $\tau$ can be expressed by the following relation:

$\tau=\pi+n \xi-\frac{\xi}{2}$,

where $n$ is a number taken from the set of numerically generated pseudo-random numbers (with the application of the MATLAB ${ }^{\circledR}$ software). Such a set of numbers is characterized by the Gaussian (normal) probability distribution. The distribution is described by the two parameters: the mean value $\mu$ and standard deviation $\sigma$. The quantity $\xi$ appearing in (14) determines the range of the values in which $n$ is closed. Additionally, we have added here the term $\frac{\xi}{2}$ which ensures that the numbers greater and smaller than $\pi$ will be chosen with equal probabilities.

By increasing the value of $\xi$, we enlarge the interval of numbers which can be randomly chosen. In consequence, the time $\tau$ becomes a subject of more substantial variations between alternate pulses and vice versa, and smaller values of $\xi$ lead to decreasing changes of $\tau$.

From the other side, a decrease in $\sigma$ leads to narrowing of the distribution. In consequence, even smaller values of $\xi$ can lead to significant changes in the values of chosen numbers. Such an effect is the result of the normalization of the probability distribution.

The influence of the changes in the values of $\xi$ and $\sigma$ on the effectiveness of the entanglement generation is shown in Fig. 6 and 7. For the all presented in this section situations, the parameters $\beta, \varepsilon, \chi_{12}$ and the deterministic part of $\tau$ are chosen in such a way that when the random perturbations are neglected a relatively weak entanglement is generated. However, when such random disturbances appear in $\tau$, they influence the negativity describing two-qubit entanglement.

In Fig. 6a, b, where maximal values of the negativity $N_{0110}$ as functions of $\xi$ are presented, the effect of random changes in the times of free evolution becomes visible. It appears that starting from the relatively weak entanglement in the system, by increasing the randomness in evolution time (increasing $\xi$ ), one can considerably increase the value of negativity. It means that the more randomness is applied to the model (to the parameter $\tau$ ), and the more benefits can be obtained from that fact. It should be emphasized that for such a case, quantum correlations become more pronounced, and thus, MES (Bell-like states) can more probably appear, despite the increase of the randomness in the value of $\tau$.
In Fig. 7a, b, the influence of the width $\sigma$ on $\max \left(N_{0110}\right)$ is shown. When cross-Kerr-type interaction is neglected, the effect of decreasing $\max \left(N_{0110}\right)$ with the increase of distribution width $\sigma$ is more pronounced. Therefore, by the proper choice of $\sigma$, we can find the best strategy to increase the entanglement in our system. We see that narrower normal distributions (the cases when random changes in the time $\tau$ are less pronounced) seem to be preferable.

From the analysis of the cases discussed here, it becomes clear that the randomness influencing the time $\tau$, and in consequence in the time of free evolution, can help in obtaining stronger entanglement in the system. This fact can especially be relevant from the experimental point of view. What is surprising, certain instabilities and randomness naturally appearing in the timings of sequences of the real laser pulses can lead to the improvement in the entanglement generation. We have shown that by the proper changes in a characteristic of such timings (including the application of random changes), one can enter the region in which the degree of achieved entanglement will be satisfactory.

\section{Summary}

We have considered the simple Bose-Hubbard system consisting of two linearly coupled nonlinear oscillators which are excited by ultra-short pulses in one or two modes. We have discussed such the model in the context of the possibility of entangled states, especially MES (Bell-type states), generation. Especially, we were interesting in finding such conditions in which our model can lead to the most effective generation of such states.

For the system discussed here, we have considered the cases when: (a) one oscillator is excited, (b) both oscillators are driven simultaneously (by synchronous and asynchronous series of pulses) and finally (c) when we apply some randomness to the timings of pulses.

We have shown that under some conditions, the evolution of our model is closed within a finite set of states. Consequently, the model considered here can be treated as a two-qubit system. Moreover, it can also be used as a good source of entangled states.

For the case when the excitations in a single mode are present, we have found the values of parameters describing our system, for which excitations in the 

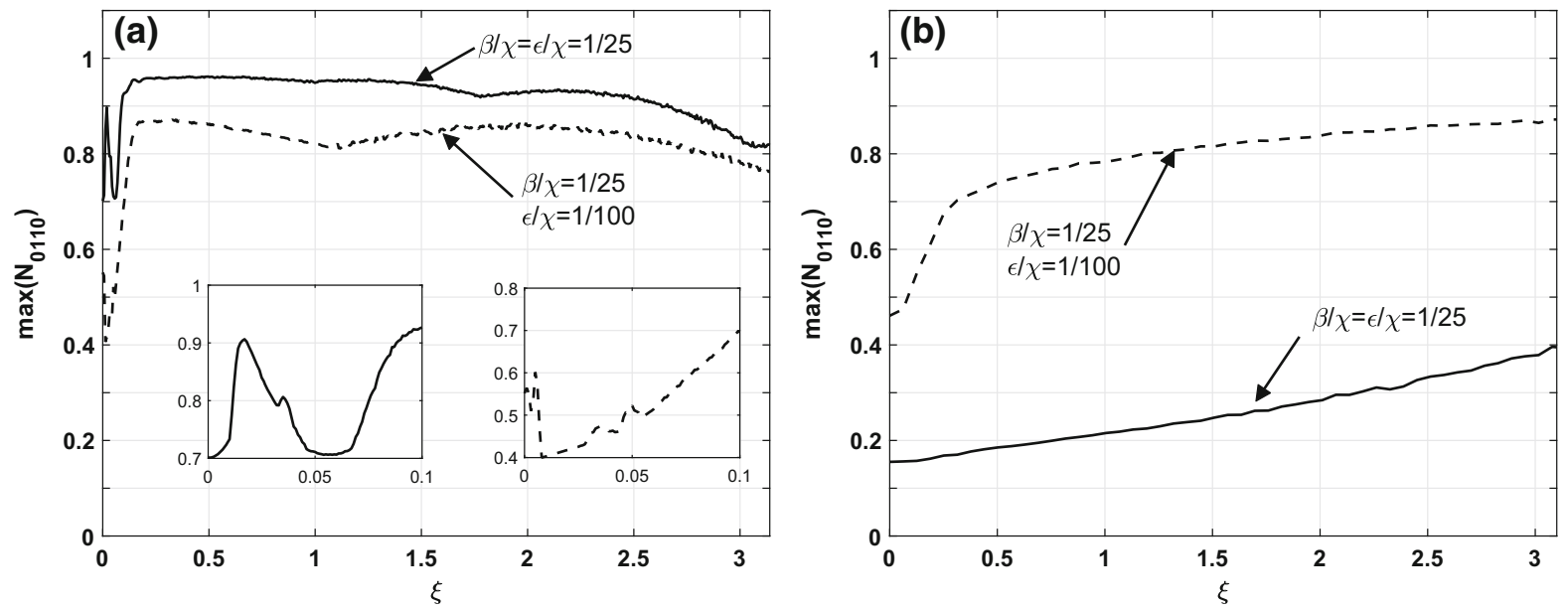

Fig. 6 Maximal achievable values of the negativity $N_{0110}$ for system with randomly disturbed free evolution's times versus $\xi:$ a $\chi_{a b}=0$; b $\chi_{a b}=1$. The remaining parameters are $\mu=0, \sigma=0.5$
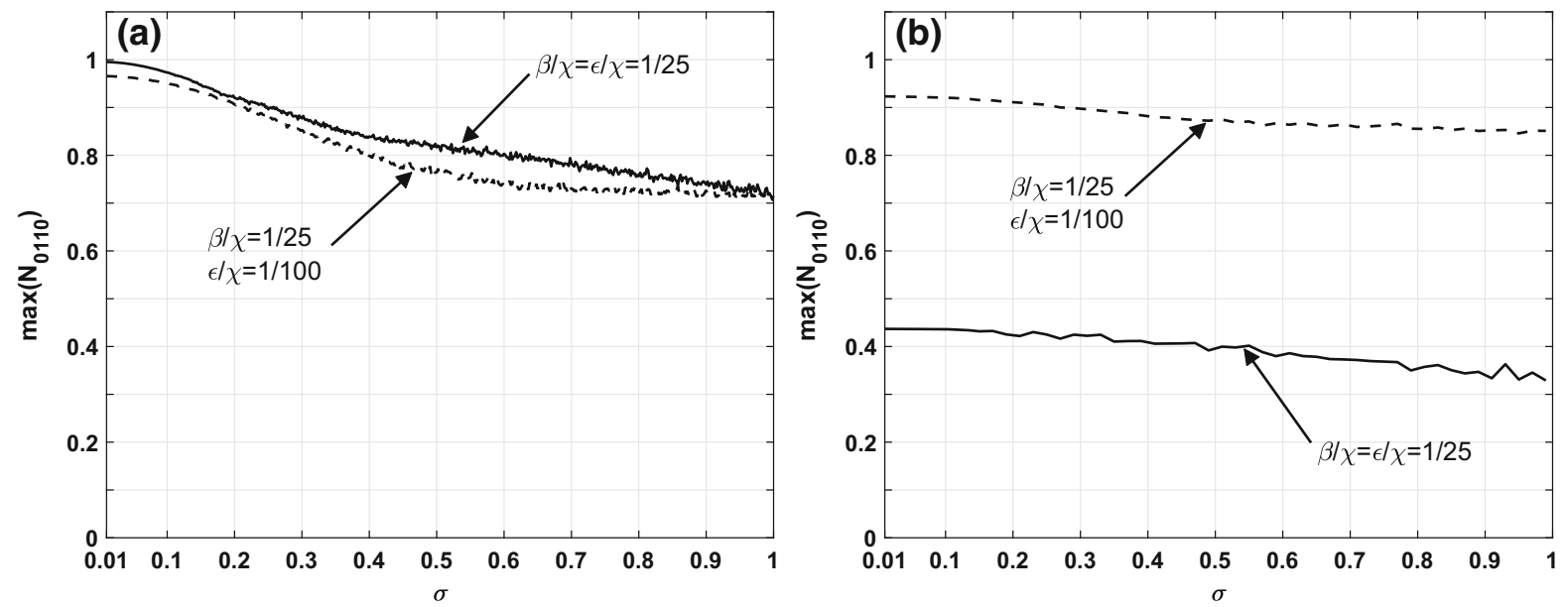

Fig. 7 Maximal achievable values of the negativity $N_{0110}$ for system with randomly disturbed free evolution's times versus $\sigma$ : a $\chi_{a b}=0 ; \mathbf{b} \chi_{a b}=1$. The remaining parameters are $\mu=0, \xi=\pi$

form of short pulses are more effective in obtaining MES than continuous driving. We have compared our results to those previously obtained and discussed in [79] where such continuous excitations were considered (it should be emphasized that in [79] only some exemplary situations corresponding to single values of the parameters characterizing the system were discussed).

We have also discussed the possibility of entanglement creation in the system excited in two modes. We have shown that the effectiveness of the generation of entangled states strongly depends on the value of the time of free evolution $T$. We have identified the ranges of $T$ for which we can treat the model as a source of MES - Bell-type states. Additionally, the same as for the model with a single-mode excitation, we have shown that when the system is simultaneously excited, there are parameters' ranges for which the system with continuous excitation is less effective as a source of entanglement than that assuming pulsed pumping.

Next, we have discussed the possibility of entanglement creation in the system excited in two modes, where the first and second modes were excited alternately. We have found such a range of the values of $\tau$ describing the time between alternate pulses for which 
the effectiveness of the generation of entangled states is very high.

Finally, we have discussed how random changes in the time between two alternate pulses influences the entanglement generation. The most important result is that even for specific range of the values of parameters when the system is not effective in the production of entangled states, it is possible to enhance the produced entanglement by an application of random changes in the time $\tau$ ( $\tau$ determines the free evolution of both oscillators between alternate pulses). We have shown that random changes of times $\tau$ may lead to the noticeable increase in the value of negativity, even if the parameters describing the system are chosen such a way that the value of the constant deterministic term in $\tau$ corresponds to the not efficient generation of the entanglement. It means that randomness can be responsible for the enhancement of quantum correlations. However, one still should remember that by not proper choice of the deterministic term of $\tau$ such process can be spoiled.

Acknowledgements J.K.K. and W.L. wish to thank the ERDF/ESF project "Nanotechnologies for Future" (CZ.02.1.01/0.0/0.0/16_019/0000754) for the financial support. J.K.K. and W.L. also acknowledge the financial support from the program of the Polish Minister of Science and Higher Education under the name "Regional Initiative of Excellence" in 2019-2022, Project No. 003/RID/2018/19, funding amount 11936 596.10 PLN.

\section{Compliance with ethical standards}

Conflict of interest The authors declare that they have no conflict of interest.

Open Access This article is distributed under the terms of the Creative Commons Attribution 4.0 International License (http:// creativecommons.org/licenses/by/4.0/), which permits unrestricted use, distribution, and reproduction in any medium, provided you give appropriate credit to the original author(s) and the source, provide a link to the Creative Commons license, and indicate if changes were made.

\section{References}

1. Ekert, A.K.: Quantum cryptography based on Bell's theorem. Phys. Rev. Lett. 67, 661-663 (1991)

2. Bennett, C.H., Wiesner, S.J.: Communication via one- and two-particle operators on Einstein-Podolsky-Rosen states. Phys. Rev. Lett. 69, 2881-2884 (1992)

3. Bennett, C.H., Brassard, G., Crépeau, C., Jozsa, R., Peres, A., Wootters, W.K.: Teleporting an unknown quantum state via dual classical and Einstein-Podolsky-Rosen channels. Phys. Rev. Lett. 70, 1895-1899 (1993)
4. Shor, P.W.: Polynomial-time algorithms for prime factorization and discrete logarithms on a quantum computer. SIAM J. Comput. 26, 1484-1509 (1997)

5. Grover, L.K.: Quantum mechanics helps in searching for a needle in a haystack. Phys. Rev. Lett. 79, 325-328 (1997)

6. Bennett, C.H., Fuchs, C.A., Smolin, J.A.: Entanglementenhanced classical communication on a noisy quantum channel. In: Hirota, O., Holevo, A.S., Caves, C.M. (eds.) Quantum Communication, Computing, and Measurement, pp. 79-88. Springer, US (1997)

7. Zheng, S.B., Guo, G.C.: Efficient scheme for two-atom entanglement and quantum information processing in cavity QED. Phys. Rev. Lett. 85, 2392-2395 (2000)

8. Nielsen, M.A., Chuang, I.L.: Quantum Computation and Quantum Information, 10th edn. Cambridge University Press, Cambridge (2010)

9. Rieffel, E.G., Polak, W.H.: Quantum Computing: A Gentle Introduction. MIT Press, Cambridge (2011)

10. Estarellas, M.P., D'Amico, I., Spiller, T.P.: Robust quantum entanglement generation and generation-plus-storage protocols with spin chains. Phys. Rev. A 95, 042335 (2017)

11. Bouwmeester, D., Pan, J.W., Mattle, K., Eible, M., Weinfurter, H., Zeilinger, A.: Experimental quantum teleportation. Nature 390, 575 (1997)

12. Özdemir, Ş.K., Bartkiewicz, K., Liu, Y.X., Miranowicz, A.: Teleportation of qubit states through dissipative channels: conditions for surpassing the no-cloning limit. Phys. Rev. A 76, 042325 (2007)

13. Cubitt, T.S., Verstraete, F., Cirac, J.I.: Entanglement flow in multipartite systems. Phys. Rev. A 71, 052308 (2005)

14. Rosenhaus, V., Smolkin, M.: Entanglement entropy flow and the ward identity. Phys. Rev. Lett. 113, 261602 (2014)

15. Shi, Jd, Wang, D., Ye, L.: Entanglement revive and information flow within the decoherent environment. Sci. Rep. 6, 30710 (2016)

16. Kumar, K.A., Reddy, J.V.R., Sugunamma, V., Sandeep, N.: Simultaneous solutions for MHD flow of Williamson fluid over a curved sheet with nonuniform heat source/sink. Heat Transf. Res. 50, 581 (2019)

17. Kumar, K.A., Sugunamma, V., Sandeep, N.: Impact of nonlinear radiation on MHD non-aligned stagnation point flow of micropolar fluid over a convective surface. J. Non Equilib. Thermodyn. 43, 327 (2018)

18. Kumar, K.A., Sugunamma, V., Sandeep, N.: Numerical exploration of MHD radiative micropolar liquid flow driven by stretching sheet with primary slip: a comparative study. J. Non Equilib. Thermodyn. 44, 101 (2018)

19. Kumar, K.A., Reddy, J.R., Sugunamma, V., Sandeep, N.: Magnetohydrodynamic Cattaneo-Christov flow past a cone and a wedge with variable heat source/sink. Alexandria Eng. J. 57(1), 435 (2018)

20. Bartkiewicz, K., Černoch, A., Chimczak, G., Lemr, K., Miranowicz, A., Nori, F.: Experimental quantum forgery of quantum optical money. npj Quantum Inf. 3, 7 (2017)

21. Pant, M., Krovi, H., Towsley, D., Tassiulas, L., Jiang, L., Basu, P., Englund, D., Guha, S.: Routing entanglement in the quantum internet. npj Quantum Inf. 5, 25 (2019)

22. Barasiński, A., Černoch, A., Lemr, K.: Demonstration of controlled quantum teleportation for discrete variables on linear optical devices. Phys. Rev. Lett. 122, 170501 (2019) 
23. Ren, J.G., Xu, P., Yong, H.L., Zhang, L., Liao, S.K., Yin, J., Liu, W.Y., Cai, W.Q., Yang, M., Li, L., Yang, K.X., Han, X., Yao, Y.Q., Li, J., Wu, H.Y., Wan, S., Liu, L., Liu, D.Q., Kuang, Y.W., He, Z.P., Shang, P., Guo, C., Zheng, R.H., Tian, K., Zhu, Z.C., Liu, N.L., Lu, C.Y., Shu, R., Chen, Y.A., Peng, C.Z., Wang, J.Y., Pan, J.W.: Ground-to-satellite quantum teleportation. Nature 549, 70 (2017)

24. Jensen, S.M.: The nonlinear coherent coupler. IEEE J. Quant. Electron. 18, 1580-1583 (1982)

25. Mă̌r, A.A.: Optical transistors and bistable devices utilizing nonlinear transmission of light in systems with undirectional coupled waves. Kvantovaya Elektronika (Moscow) 9, 2302-2996 (1982). [Sov J Quantum Electron, 12, 14901494 (1982)]

26. Kowalewska-Kudłaszyk, A., Leoński, W.: Finitedimensional states and entanglement generation for a nonlinear coupler. Phys. Rev. A 73, 042318 (2006)

27. Olsen, M.K.: Bright entanglement in the intracavity nonlinear coupler. Phys. Rev. A 73, 053806 (2006)

28. Kowalewska-Kudłaszyk, A., Leoński, W., Peřina Jr., J.: Photon-number entangled states generated in Kerr media with optical parametric pumping. Phys. Rev. A 83, 052326 (2011)

29. Horák, R., Bertolotti, M., Sibilia, C., Peřina, J.: Quantum effects in a nonlinear coherent coupler. J. Opt. Soc. Am. B 6(2), 199-204 (1989)

30. Korolkova, N., Peřina, J.: Quantum statistics and dynamics of Kerr nonlinear couplers. Opt. Commun. 136, 135-149 (1996)

31. Korolkova, N., Peřina, J.: Kerr nonlinear coupler with varying linear coupling coefficient. J. Mod. Opt. 44, 1525-1534 (1997)

32. Fiurášek, J., Křepelka, J., Peřina, J.: Quantum-phase properties of the Kerr couplers. Opt. Commun. 167, 115 (1999)

33. Ibrahim, A.B.M.A., Umarov, B.A., Wahiddin, M.R.B.: Squeezing in the Kerr nonlinear coupler via phase-space representation. Phys. Rev. A 61, 043804 (2000)

34. Julius, R., Ibrahim, A.B.M.: Quantum squeezing in multichannel nonlinear coupler. J. Comput. Sci. Comput. Math. 7(4), 131-136 (2017)

35. Miranowicz, A., Leoński, W.: Two-mode optical state truncation and generation of maximally entangled states in pumped nonlinear couplers. J. Phys. B At. Mol. Opt. Phys. 39, 1683 (2006)

36. Kowalewska-Kudłaszyk, A., Leoński, W., Peřina Jr., J.: Generalized Bell states generation in a parametrically excited nonlinear coupler. Physica Scripta 2012(T147), 014016 (2012)

37. Kowalewska-Kudłaszyk, A.: Entanglement in a nonlinear coupler: the cross-action effect. Physica Scripta 2013(T153), 014039 (2013)

38. Kowalewska-Kudłaszyk, A., Leoński, W.: Nonlinear coupler operating on Werner-like states; entanglement creation, its enhancement, and preservation. J. Opt. Soc. Am. B 31(6), 1290-1297 (2014)

39. Zhang, M., Helmerson, K., You, L.: Entanglement and spin squeezing of Bose-Einstein-condensed atoms. Phys. Rev. A 68, 043622 (2003)

40. Gerry, C.C., Campos, R.A.: Generation of maximally entangled states of a Bose-Einstein condensate and Heisenberglimited phase resolution. Phys. Rev. A 68, 025602 (2003)
41. Vidal, J., Palacios, G., Aslangul, C.: Entanglement dynamics in the Lipkin-Meshkov-Glick model. Phys. Rev. A 70, 062304 (2004)

42. Loss, D., DiVincenzo, D.P.: Quantum computation with quantum dots. Phys. Rev. A 57, 120-126 (1998)

43. Miranowicz, A., Özdemir, Ş.K., Liu, Yx, Koashi, M., Imoto, N., Hirayama, Y.: Generation of maximum spin entanglement induced by a cavity field in quantum-dot systems. Phys. Rev. A 65, 062321 (2002)

44. Cirac, J.I., Zoller, P.: Quantum computations with cold trapped ions. Phys. Rev. Lett. 74, 4091-4094 (1995)

45. Lougovski, P., Solano, E., Walther, H.: Generation and purification of maximally entangled atomic states in optical cavities. Phys. Rev. A 71, 013811 (2005)

46. Qin, W., Miranowicz, A., Li, P.B., Lü, X.Y., You, J.Q., Nori, F.: Exponentially enhanced light-matter interaction, cooperativities, and steady-state entanglement using parametric amplification. Phys. Rev. Lett. 120, 093601 (2018)

47. Alexanian, M.: Dynamical generation of maximally entangled states in two identical cavities. Phys. Rev. A 84, 052302 (2011)

48. Almutairi, K., Tanaś, R., Ficek, Z.: Generating two-photon entangled states in a driven two-atom system. Phys. Rev. A 84, 013831 (2011)

49. Owen, E.T., Dean, M.C., Barnes, C.H.W.: Generation of entanglement between qubits in a one-dimensional harmonic oscillator. Phys. Rev. A 85, 022319 (2012)

50. Brida, G., Chekhova, M., Genovese, M., Krivitsky, L.: Generation of different Bell states within the spontaneous parametric down-conversion phase-matching bandwidth. Phys. Rev. A 76, 053807 (2007)

51. Coto, R., Orszag, M., Eremeev, V.: Generation and protection of a maximally entangled state between many modes in an optical network with dissipation. Phys. Rev. A 93, 062302 (2016)

52. Kalaga, J.K., Kowalewska-Kudłaszyk, A., Leoński, W., Barasiński, A.: Quantum correlations and entanglement in a model comprised of a short chain of nonlinear oscillators. Phys. Rev. A 94, 032304 (2016)

53. Leoński, W., Kowalewska-Kudłaszyk, A.: Quantum scissors - finite-dimensional states engineering. In: Wolf, E. (ed.) Progress in Optics, vol. 56, pp. 131-185. Elsevier, Amsterdam (2011)

54. Birnbaum, K.M., Boca, A., Miller, R., Boozer, A.D., Northup, T.E., Kimble, H.J.: Photon blockade in an optical cavity with one trapped atom. Nature 436, 87 (2005)

55. Faraon, A., Fushman, I., Englund, D., Stoltz, N., Petroff, P., Vučković, J.: Coherent generation of non-classical light on a chip via photon-induced tunnelling and blockade. Nat. Phys. 4, 859 (2008)

56. Bretheau, I., Campagne-Ibercq, P., Flurin, E., Mallet, F., Huard, B.: Quantum dynamics of an electromagnetic mode that cannot contain N photons. Science 348, 776 (2015)

57. Tanaś, R., Miranowicz, A., Kielich, S.: Squeezing and its graphical representations in the anharmonic oscillator model. Phys. Rev. A 43, 4014-4021 (1991)

58. Wilson-Gordon, A.D., Buzek, V., Knight, P.L.: Statistical and phase properties of displaced Kerr states. Phys. Rev. A 44, 7647-7656 (1991)

59. Paprzycka, M., Tanaś, R.: Discrete superpositions of coherent states and phase properties of the m-photon anharmonic 
oscillator. Quantum Opt. J. Eur. Opt. Soc. Part B 4, 331 (1992)

60. Gerry, C.C., Grobe, R.: Statistical properties of squeezed Kerr states. Phys. Rev. A 49, 2033-2039 (1994)

61. Peřinová, V., Vrana, V., Lukš, A., Křepelka, J.: Quantum statistics of displaced Kerr states. Phys. Rev. A 51, 24992515 (1995)

62. Tanaś, R., Miranowicz, A., Gantsog, T.: VI quantum phase properties of nonlinear optical phenomena. In: Wolf, E. (ed.) Progress in Optics 35, pp. 355-446. Elsevier, Amsterdam (1996)

63. Leoński, W., Tanaś, R.: Possibility of producing the onephoton state in a kicked cavity with a nonlinear Kerr medium. Phys. Rev. A 49, R20-R23 (1994)

64. Leoński, W.: Finite-dimensional coherent-state generation and quantum-optical nonlinear oscillator models. Phys. Rev. A. 55, 3874-3878 (1997)

65. Leoński, W.: Fock-states in a Kerr medium with parametric pumping. Phys. Rev. A 54, 3369-3372 (1996)

66. Leoński, W.: Finite energy states generation by periodically pulsed nonlinear oscillator. J. Mod. Opt. 48, 877 (2001)

67. Saitô, N., Hirooka, H., Ooyama, N.: Computer studies of ergodicity in coupled anharmonic oscillators. J. Phys. Soc. Jpn. S26, 233 (1969)

68. Saitô, N., Hirooka, H., Ooyama, N.: Discussion of computer studies of ergodicity in coupled anharmonic oscillators. J. Phys. Soc. Jpn. S26, 234 (1969)

69. Milburn, G.J.: Quantum and classical Liouville dynamics of the anharmonic oscillator. Phys. Rev. A 33, 674-685 (1986)

70. Leoński, W.: Quantum and classical dynamics for a pulsed nonlinear oscillator. Phys. A 233, 365-378 (1996)

71. Milburn, G.J.: Coherence and chaos in a quantum optical system. Phys. Rev. A 41, 6567(R) (1990)

72. Milburn, G.J., Holmes, C.A.: Quantum coherence and classical chaos in a pulsed parametric oscillator with a Kerr nonlinearity. Phys. Rev. A 44(7), 4704-4711 (1991)

73. Adamyan, H.H., Manvelyan, S.B., Kryuchkyan, G.Y.: Chaos in a double driven dissipative nonlinear oscillator. Phys. Rev. E 64, 046219 (2001)

74. Kowalewska-Kudłaszyk, A., Kalaga, J.K., Leoński, W.: Wigner-function nonclassicality as indicator of quantum chaos. Phys. Rev. E 78, 066219 (2008)

75. Kowalewska-Kudłaszyk, A., Kalaga, J.K., Leoński, W.: Long-time fidelity and chaos for a kicked nonlinear oscillator system. Phys. Lett. A 373(15), 1334-1340 (2009)
76. Gevorgyan, T.V., Shahinyan, A.R., Kryuchkyan, G.Y.: Quantum interference and sub-Poissonian statistics for timemodulated driven dissipative nonlinear oscillators. Phys. Rev. A 79, 053828 (2009)

77. Kowalewska-Kudłaszyk, A., Kalaga, J.K., Leoński, W., Cao Long, V.: Kullback-Leibler quantum divergence as an indicator of quantum chaos. Phys. Lett. A 376, 1280-1286 (2012)

78. Kalaga, J.K., Leoński, W., Szczȩśniak, R.: Quantum steering in an asymmetric chain of nonlinear oscillators. Photonics Lett. Pol. 9, 97-99 (2017)

79. Kowalewska-Kudłaszyk, A., Leoński, W., Nguyen, T.D., Long, V.C.: Kicked nonlinear quantum scissors and entanglement generation. Phys. Scr. T160, 014023 (2014)

80. Schmidt, H., Imamoglu, A.: Giant Kerr nonlinearities obtained by electromagnetically induced transparency. Opt. Lett. 21, 1936-1938 (1996)

81. Peres, A.: Separability criterion for density matrices. Phys. Rev. Lett. 77, 1413-1415 (1996)

82. Horodecki, M., Horodecki, P., Horodecki, R.: Separability of mixed states: necessary and sufficient conditions. Phys. Lett. A 223, 1-8 (1996)

83. Paschotta, R.: Article on 'Timing Jitter' in Encyclopedia of Laser Physics and Technology. Wiley, Weinheim (2008)

84. Paschotta, R.: Noise of mode-locked lasers. Part I: numerical model. Appl. Phys. 79, 153 (2004)

85. Paschotta, R.: Noise of mode-locked lasers. Part II: timing jitter and other fluctuations. Appl. Phys. 79, 163 (2004)

86. Paschotta, R.: Timing jitter and phase noise of mode-locked fiber lasers. Opt. Express 18, 5041 (2010)

Publisher's Note Springer Nature remains neutral with regard to jurisdictional claims in published maps and institutional affiliations. 